\section{Prevalência e fatores associados a porte de arma e envolvimento em agressão física entre adolescentes de 15 a 18 anos: estudo de base populacional}

\author{
Prevalence of weapons possession and associated \\ factors and involvement in physical aggression \\ among adolescents 15 to 18 years of age: \\ a population-based study
}

1 Universidade Católica de Pelotas, Pelotas, Brasil. 2 Universidade Federal de Pelotas, Pelotas, Brasil.

Correspondência R. A. Silva

Escola de Psicologia Universidade Católica de Pelotas.

Rua Almirante Barroso

1202, sala G-109, Pelotas, RS 96020-380, Brasil.

ricardo.as@uol.com.br

\begin{abstract}
This cross-sectional, population-based study aimed to evaluate the prevalence of weapons possession and associated factors and involvement in physical aggression among adolescents 15 to 18 years of age $(n=960)$ in the city of Pelotas, Rio Grande do Sul State, Brazil. Ninety of the city's 448 census tracts were selected, and 86 houses in each tract were visited. The statistical analysis used Poisson regression. Prevalence rates in the sample were $22.8 \%$ for involvement in fights with physical aggression and $9.6 \%$ for weapons possession in the previous 12 months. The study concluded that young males that use alcohol and/or illegal drugs and present minor psychiatric disorders show a higher probability of weapons possession and involvement in physical fights.
\end{abstract}

Weapons; Agrression; Violence; Adolescent
Ricardo Azevedo da Silva 1

Karen Jansen 1

Russélia Vanila Godoy 1

Luciano Dias Mattos Souza 1

Bernardo Lessa Horta 2

Ricardo Tavares Pinheiro 1

A adolescência é o período da vida em que o jovem está exposto a vários comportamentos que podem comprometer sua saúde física e mental 1 . Em geral, tais atitudes estão ligadas ao caráter exploratório dessa etapa do desenvolvimento, porém podem levar à consolidação desses comportamentos com significativas conseqüências negativas nos níveis individual, familiar e social 1 . O grupo etário de 10 a 24 anos é o segmento populacional em que as causas externas adquirem sua máxima expressão (Yunes \& Rajs, 1994, apud Sauer \& Wagner 2). Evidências apontam a magnitude e o crescimento da mortalidade devido a causas externas nos adolescentes por homicídios perpetrados por arma de fogo, sobretudo naqueles do gênero masculino ${ }^{3}$.

Alguns comportamentos agressivos como portar armas e participar de brigas apresentam alta prevalência entre os jovens, e ainda mostram-se associados com altos índices de homicídio e suicídio 4,5. Em 2007, uma investigação conduzida por Swahn \& Bossarte 6 aponta uma associação significativa entre brigar e portar armas com ideação suicida e tentativa de suicídio 7.

A literatura aponta associação de atitudes anti-sociais em adolescentes com déficit intelectual ${ }^{8}$, uso de medicamentos ${ }^{8}$, álcool ${ }^{9}$, drogas ${ }^{9}$, dificuldades na relação parental 8,9,10,11, abuso infantil ${ }^{11}$, baixo nível sócio-econômico ${ }^{8}$, família 
numerosa, poucos amigos e baixo rendimento escolar 12 .

A presente investigação buscou avaliar a prevalência e os fatores associados a porte de arma e envolvimento em agressão física entre adolescentes de 15 a 18 anos, por meio de um estudo de base populacional realizado na cidade de Pelotas, Rio Grande do Sul, no extremo Sul do Brasil.

\section{Método}

Estudo transversal de base populacional em uma amostra representativa de adolescentes com idades entre 15 e 18 anos, residentes na zona urbana da cidade de Pelotas (345 mil habitantes), no extremo Sul do Brasil, no ano de 2002 (Instituto Brasileiro de Geografia e Estatística. http:// www.ibge.gov.br). A investigação foi aprovada pelo comitê de ética em pesquisa da Universidade Católica de Pelotas (UCPel) e finaciada pela Fundação de Amparo à Pesquisa do Estado do Rio Grande do Sul (FAPERGS), com o protocolo $n^{\circ}$. 01/1552.9. Foi assegurado o direito a não participação, à confidencialidade dos dados e ao cuidado na utilização das informações nos trabalhos escritos, de modo que os participantes não possam ser identificados. Os adolescentes que tiveram indicativo para transtornos mentais comuns receberam encaminhamento para atendimento no ambulatório de psiquiatria do hospital universitário da UCPel.

A partir dos 448 setores censitários da zona urbana da cidade de Pelotas, 90 setores foram escolhidos aleatoriamente e nestes foram sorteados um quarteirão e uma esquina como ponto inicial, a partir do qual 86 residências foram sistematicamente visitadas. No total, 7.740 domicílios foram visitados pela equipe de pesquisa. O tamanho da amostra foi calculado para um estudo amplo sobre comportamentos de saúde em adolescentes. Em função dos seus múltiplos objetivos, o tamanho da amostra calculado foi de 800 adolescentes.

Após a obtenção do consentimento por escrito dos pais ou responsável pelo adolescente, o mesmo respondia a um questionário auto-aplicado e sigiloso com questões sobre nível sócioeconômico, estudo, trabalho, prática religiosa, uso de drogas ilícitas, tabagismo, consumo de álcool e ocorrência de transtornos mentais comuns. Após o adolescente responder ao questionário, este era depositado em uma urna lacrada, com a finalidade de manter o sigilo.

Para avaliar o nível sócio-econômico, utilizou-se a classificação da Associação Brasileira de Institutos de Pesquisa de Mercado (ABIPEME) 13. Essa classificação é baseada na acumulação de bens materiais e na escolaridade do chefe da família, e classifica os sujeitos em cinco classes (A, B, C, D e E) de acordo com o escore obtido.

Transtornos mentais comuns foram avaliados usando-se o Self-Report Questionnaire (SRQ20), produzido pela Organização Mundial da Saúde e validado por Mari et al. 14. O instrumento consiste em 20 questões de sim ou não (4 sobre sintomas físicos e 16 sobre transtornos psicológicos nos últimos trinta dias), sendo que o ponto de corte utilizadado foi o proposto na validação para o Brasil, 6 para homens e 7 para mulheres. Transtornos mentais comuns é uma categoria criada por Goldberg \& Huxley 15, e inclui sintomas não psicóticos como depressão, ansiedade e transtornos somatoformes.

No que diz respeito ao comportamento agressivo, considerou-se separadamente duas variáveis dicotomizadas como desfechos. Essas foram coletadas em relação aos últimos 12 meses: ter portado alguma vez uma arma - revólver, faca ou canivete - e ter participado de briga com agressão física.

Realizou-se duas digitações no programa Epi Info 6.04d (Centers for Disease Control and Prevention, Atlanta, Estados Unidos) e estas foram comparadas para que as inconsistências fossem solucionadas. Foi realizada análise bruta usando-se o teste qui-quadrado e análise ajustada (multivariada) por meio de regressão de Poisson com ajuste robusto da variância e correção para efeitos de delineamento, no programa estatístico Stata 9 (Stata Corp., College Station, Estados Unidos). A regressão de Poisson foi usada tendo em vista a elevada prevalência dos desfechos, pois a utilização de regressão logística levaria à superestimação da razão de prevalências 16 .

A análise multivariada foi realizada seguindo um modelo hierárquico de base teórica, em que as variáveis foram agrupadas em dois níveis. Após a análise bruta, as variáveis com um valor de p 0,20 no teste de razões de verossimilhança permaneceram no modelo. Nesse tipo de análise, as variáveis situadas no primeiro nível hierárquico foram consideradas como potenciais confundidores da relação entre estas e os desfechos: porte de arma e briga com agressão física nos últimos 12 meses. Na análise ajustada, as variáveis com $\mathrm{p} \leq$ 0,05 foram consideradas como fatores associados com os comportamentos agressivos estudados.

Para o desfecho porte de arma nos últimos 12 meses, o modelo hierárquico foi composto no primeiro nível por gênero, nível sócio-econômico, idade, estudo, morar com os pais, trabalho remunerado e prática religiosa. No segundo nível, foram incluídas as variáveis uso de drogas ilícitas, álcool, tabaco e presença de transtornos 
mentais comuns. Na análise do desfecho briga com agressão física, o mesmo modelo foi utilizado acrescentando-se a variável porte de arma nos últimos 12 meses.

\section{Resultados}

A investigação avaliou uma amostra representativa de 960 adolescentes com idades entre 15 e 18 anos, residentes na zona urbana da cidade de Pelotas. Dos 1.039 adolescentes identificados, as perdas ou recusas representaram 7,6\% (79) da amostra e ocorreram porque o adolescente não foi encontrado em sua residência após pelo menos três tentativas, ou não houve a concordância dos pais ou responsáveis com a realização da entrevista.

A população estudada situa-se, preponderantemente, no nível sócio-econômico C $(38,1 \%)$, na média etária de 16,5 anos de idade e apresentava nove anos ou mais de estudo (58\%). A maioria relatou escolaridade paterna e materna inferior a oito anos de estudo, assim como residir com pai e mãe e não praticar religião. Dos jovens entrevistados, $21 \%$ possuem trabalho remunerado. Com relação ao uso de substâncias, 43,2\%, 16,4\% e $8,6 \%$ afirmaram ter consumido, respectivamente, bebida alcoólica, tabaco e drogas ilícitas nos últimos trinta dias. Além disso, o sedentarismo foi identificado em 374 adolescentes. A prevalência de transtornos mentais comuns nos entrevistados foi de $28,9 \%$. No que se refere ao porte de arma de fogo ou branca nos últimos $12 \mathrm{me}$ ses, 9,6\% dos adolescentes relataram tê-lo feito, enquanto que $22,8 \%$ afirmaram ter participado de pelo menos uma briga com agressão física no mesmo período.

$\mathrm{Na}$ análise bruta, o desfecho porte de arma nos últimos 12 meses esteve associado com gênero, estar estudando no momento da entrevista, possuir trabalho remunerado, praticar alguma religião, assim como consumir drogas ilícitas, álcool e/ou tabaco no útlimo mês. Após o ajuste por meio do modelo hierárquico proposto, porte de arma no último ano manteve associação signficativa com gênero, estar estudando, possuir trabalho remunerado e uso de drogas ilícitas e bebida alcoólica no último mês. Passaram a apresentar associação com o desfecho, a presença de transtornos mentais comuns e nível sócio-econômico. Perderam significância estatística a prática religiosa e o consumo de tabaco (Tabela 1).

Os meninos mostram 3,6 vezes maior probabilidade de portar armas do que as meninas. Trabalhar de forma remunerada aumenta em 97\% a probabilidade de apresentar esse comportamento em relação aos que não trabalham.
Estar estudando incrementa a probabilidade em $74 \%$. O uso de álcool e drogas ilícitas no último mês aumenta, respectivamente, em $77 \%$ e $69 \%$. A presença de transtornos mentais comuns acrescenta $69 \%$ a probabilidade de portar armas nos últimos 12 meses (Tabela 1).

No que se refere ao envolvimento em brigas com agressão física nos últimos 12 meses, inicialmente mostraram associação significativa com tal desfecho as variáveis gênero, trabalho remunerado, prática religiosa, consumo de drogas ilícitas, álcool e/ou tabaco no último mês, bem como porte de arma no último ano. Entretanto, as variáveis nível sócio-econômico, idade, estudo atual e morar com pai e mãe não foram incluídas na análise ajustada, uma vez que apresentaram p > 0,20. (Tabela 2).

Após a análise ajustada, ter participado de briga com agressão física nos últimos 12 meses manteve-se associada com as variáveis gênero, uso de drogas ilícitas e álcool no último mês e porte de arma nos últimos 12 meses. A relação entre a presença de transtornos mentais comuns e o desfecho passou a ser estatisticamente significativa, entretanto, trabalho remunerado, prática religiosa e consumo de tabaco perderam significância estatística (Tabela 2).

Os adolescentes do gênero masculino têm aproximadamente 2,2 vezes maior probabilidade de se envolver em briga com agressão no último ano em relação às meninas. O uso de drogas ilícitas aumenta em $48 \%$ e beber no último mês aumenta em $78 \%$ a probabilidade para esse comportamento agressivo. Os participantes que possuem um indicativo de transtornos mentais comuns têm $31 \%$ maior probabilidade de ter participado de brigas com agressão no último ano. Os comportamentos agressivos investigados no presente estudo também apresentaram associação entre si após o controle estatístico. Os indivíduos que portaram arma nos últimos 12 meses apresentaram 2,1 vezes maior probabilidade de ter participado de brigas com agressão física no mesmo período (Tabela 2).

\section{Discussão}

O comportamento violento faz parte do processo de desenvolvimento humano, a agressão física é uma forma comum de violência interpessoal entre os adolescentes de diferentes culturas. Estima-se que 31 a 50\% dos adolescentes participam de brigas com agressão física 17,18 e que 5\% a $34 \%$ dos adolescentes relataram portar algum tipo de arma 19,20. A prevalência desses comportamentos na presente amostra, apesar de elevada $(22,8 \%$ participaram de brigas com agressão 
Tabela 1

Porte de arma nos últimos 12 meses.

\begin{tabular}{|c|c|c|c|c|c|c|}
\hline Variáveis & $\begin{array}{l}\text { Distribuição da } \\
\text { amostra }\end{array}$ & $\begin{array}{c}\text { Prevalência do } \\
\text { desfecho } \\
\%(n)\end{array}$ & $\begin{array}{l}\text { RP Bruta } \\
\text { (IC95\%) }\end{array}$ & Valor de $p$ & $\begin{array}{l}\text { RP ajustada } \\
\text { (IC95\%) }\end{array}$ & Valor de $p$ \\
\hline \multicolumn{7}{|l|}{ Primeiro nível hierárquico } \\
\hline Gênero & & & & 0,000 & & 0,000 \\
\hline Masculino & 463 & $15,8(72)$ & $4,10(2,51-6,68)$ & & $3,66(2,19-6,11)$ & \\
\hline Feminino & 497 & 3,9 (19) & 1,00 & & 1,00 & \\
\hline Nível sócio-econômico & & & & & & 0,014 * \\
\hline$A+B$ & 346 & $11,8(41)$ & $1,60(0,94-2,72)$ & 0,065 * & $1,83(1,11-3,03)$ & \\
\hline C & 366 & $8,9(32)$ & $1,21(0,69-2,10)$ & & $1,23(0,72-2,10)$ & \\
\hline$D+E$ & 248 & $7,4(18)$ & 1,00 & & 1,00 & \\
\hline Idade (anos) & & & & 0,118 & & 0,427 \\
\hline $15-16$ & 498 & $8,2(40)$ & 1,00 & & 1,00 & \\
\hline $17-18$ & 462 & $11,2(51)$ & $1,37(0,92-2,03)$ & & $1,17(0,79-1,73)$ & \\
\hline Estuda atualmente & & & & 0,023 & & 0,009 \\
\hline Sim & 801 & $8,6(68)$ & 1,00 & & 1,00 & \\
\hline Não & 159 & $14,8(23)$ & $1,73(1,11-2,68)$ & & $1,74(1,14-2,66)$ & \\
\hline Mora com pai e mãe & & & & 0,165 & & 0,076 \\
\hline Sim & 583 & 8,5 (49) & 1,00 & & 1,00 & \\
\hline Não & 377 & $11,4(42)$ & $1,35(0,91-1,99)$ & & $1,41(0,96-2,06)$ & \\
\hline Trabalho remunerado & & & & 0,000 & & 0,001 \\
\hline Sim & 202 & $18,1(36)$ & $2,46(1,67-3,63)$ & & $1,97(1,31-2,95)$ & \\
\hline Não & 758 & $7,4(55)$ & 1,00 & & 1,00 & \\
\hline Pratica religião & & & & 0,037 & & 0,512 \\
\hline Sim & 343 & $6,8(23)$ & 1,00 & & 1,00 & \\
\hline Não & 617 & $11,2(68)$ & $1,65(1,05-2,59)$ & & $1,17(0,75-1,84)$ & \\
\hline \multicolumn{7}{|l|}{ Segundo nível hierárquico } \\
\hline Consumo de drogas ilícitas & & & & 0,000 & & 0,016 \\
\hline Sim & 83 & $23,8(19)$ & $2,86(1,82-4,49)$ & & $1,69(1,10-2,60)$ & \\
\hline Não & 877 & $8,3(72)$ & 1,00 & & 1,00 & \\
\hline Consumo de bebida alcoólica & & & & 0,000 & & 0,011 \\
\hline Sim & 410 & $15,0(61)$ & $2,65(1,75-4,03)$ & & $1,77(1,14-2,75)$ & \\
\hline Não & 538 & $5,6(30)$ & 1,00 & & 1,00 & \\
\hline Consumo de tabaco & & & & 0,000 & & 0,056 \\
\hline $\operatorname{Sim}$ & 157 & $18,2(28)$ & $2,29(1,52-3,45)$ & & $1,52(0,99-2,35)$ & \\
\hline Não & 803 & $7,9(63)$ & 1,00 & & 1,00 & \\
\hline $\begin{array}{l}\text { Transtornos psiquiátricos } \\
\text { menores }\end{array}$ & & & & 0,121 & & 0,005 \\
\hline Sim & 277 & $12,1(33)$ & $1,41(0,94-2,11)$ & & $1,69(1,17-2,45)$ & \\
\hline Não & 683 & $8,6(58)$ & 1,00 & & 1,00 & \\
\hline Total & 960 & $9,6(91)$ & & & & \\
\hline
\end{tabular}

RP: razão de prevalência; IC95\%: intervalo de 95\% de confiança.

* Valor de p com teste de linearidade. 
Tabela 2

Brigas com agressão física nos últimos 12 meses.

\begin{tabular}{|c|c|c|c|c|c|c|}
\hline Variáveis & $\begin{array}{l}\text { Distribuição da } \\
\text { amostra }\end{array}$ & $\begin{array}{c}\text { Prevalência do } \\
\text { desfecho } \\
\%(n)\end{array}$ & $\begin{array}{l}\text { RP Bruta } \\
\text { (IC95\%) }\end{array}$ & Valor de p & $\begin{array}{l}\text { RP ajustada } \\
\text { (IC95\%) }\end{array}$ & Valor de $p$ \\
\hline \multicolumn{7}{|l|}{ Primeiro nível hierárquico } \\
\hline Gênero & & & & 0,000 & & 0,000 \\
\hline Masculino & 463 & 32,7 (149) & $2,38(1,84-3,08)$ & & $2,23(1,70-2,91)$ & \\
\hline Feminino & 497 & $13,7(68)$ & 1,00 & & 1,00 & \\
\hline Nível sócio-econômico & & & & 0,566 * & & \\
\hline$A+B$ & 346 & $22,3(77)$ & 1,00 & & - & - \\
\hline C & 366 & $22,3(80)$ & $1,00(0,76-1,32)$ & & - & - \\
\hline$D+E$ & 248 & $24,4(60)$ & $1,10(0,82-1,47)$ & & - & - \\
\hline Idade (anos) & & & & 0,679 & & \\
\hline $15-16$ & 498 & $22,2(110)$ & 1,00 & & - & - \\
\hline $17-18$ & 462 & 23,5 (107) & $1,06(0,84-1,34)$ & & - & - \\
\hline Estuda atualmente & & & & 0,727 & & \\
\hline Sim & 801 & 22,5 (179) & 1,00 & & - & - \\
\hline Não & 159 & $24,2(38)$ & $1,07(0,79-1,46)$ & & - & - \\
\hline Mora com o pai e a mãe & & & & 0,273 & & \\
\hline Sim & 583 & $21,5(124)$ & 1,00 & & - & - \\
\hline Não & 377 & $24,8(93)$ & $1,15(0,91-1,46)$ & & - & - \\
\hline Trabalho remunerado & & & & 0,006 & & 0,060 \\
\hline Sim & 202 & $30,5(60)$ & $1,46(1,14-1,88)$ & & $1,27(0,99-1,64)$ & \\
\hline Não & 758 & $20,8(157)$ & 1,00 & & 1,00 & \\
\hline Pratica religião & & & & 0,010 & & 0,205 \\
\hline Sim & 343 & $17,9(61)$ & 1,00 & & & \\
\hline Não & 617 & $25,5(156)$ & $1,42(1,09-1,85)$ & & $1,19(0,91-1,55)$ & \\
\hline \multicolumn{7}{|l|}{ Segundo nível hierárquico } \\
\hline Consumo de drogas ilícitas & & & & 0,000 & & 0,005 \\
\hline Sim & 83 & $46,9(38)$ & $2,28(1,75-2,97)$ & & $1,48(1,12-1,95)$ & \\
\hline Não & 877 & $20,6(179)$ & 1,00 & & 1,00 & \\
\hline Consumo de bebida alcoólica & & & & 0,000 & & 0,000 \\
\hline Sim & 410 & $33,8(137)$ & $2,30(1,80-2,93)$ & & $1,78(1,37-2,32)$ & \\
\hline Não & 538 & $14,7(79)$ & 1,00 & & 1,00 & \\
\hline Consumo de tabaco & & & & 0,001 & & 0,495 \\
\hline Sim & 157 & $33,5(52)$ & $1,62(1,25-2,10)$ & & $1,10(0,84-1,44)$ & \\
\hline Não & 803 & $20,7(165)$ & 1,00 & & 1,00 & \\
\hline $\begin{array}{l}\text { Transtornos psiquiátricos } \\
\text { menores }\end{array}$ & & & & 0,097 & & 0,022 \\
\hline $\operatorname{Sim}$ & 277 & $26,5(73)$ & $1,25(0,98-1,59)$ & & $1,31(1,04-1,66)$ & \\
\hline Não & 683 & $21,3(144)$ & 1,00 & & 1,00 & \\
\hline Porte de arma & & & & 0,000 & & 0,000 \\
\hline Sim & 90 & $63,3(57)$ & $3,43(2,77-4,24)$ & & $2,09(1,64-2,68)$ & \\
\hline Não & 850 & $18,5(157)$ & 1,00 & & 1,00 & \\
\hline Total & 960 & $22,8(217)$ & - & - & - & - \\
\hline
\end{tabular}

RP: razão de prevalência; IC95\%: intervalo de 95\% de confiança.

* Valor de p com teste de linearidade. 
e 9,6\% portaram armas), é inferior à média dos demais estudos sobre comportamento agressivo com adolescentes.

Os fatores associados a comportamentos violentos podem ser encontrados no indivíduo, no ambiente ou na capacidade individual de responder às demandas do ambiente 18 .

Neste estudo, ser do sexo masculino aumenta o risco para ambos os desfechos estudados, assim como em todos os demais artigos que investigaram a questão de gênero 16,17,18,19,20,21, $22,23,24,25$. Os achados sugerem que meninos são os que mais usam a violência para resolver seus conflitos interpessoais. Esse resultado reflete normas sociais e culturais da nossa sociedade, na qual brigar é um comportamento classificado como aceitável entre meninos.

$\mathrm{Na}$ amostra em estudo, os jovens que possuem um trabalho remunerado são mais propensos a portar armas brancas ou de fogo. Não foram localizados outros estudos que avaliaram a influência de possuir uma ocupação relacionando-a com envolvimento em comportamentos agressivos. Esse é um dado significativo, pois é contrário à hipótese de que uma maior inserção social inibe tais comportamentos. Contudo, é possível formular a hipótese de que o trabalho nessa idade está ligado à necessidade de sobrevivência e não à satisfação profissional, pois segundo a análise da Pesquisa Nacional por Amostra de Domicílios (PNAD 2005), 68,1\% dos jovens trabalhadores até 19 anos recebem menos de um salário mínimo 26. Esses jovens, provavelmente, estão buscando a satisfação de necessidades imediatas de sobrevivência e frustrados com os resultados obtidos.

Tal suposição pode ser reforçada pelo dado a seguir devido ao fato de que o abandono do estudo também está relacionado ao trabalho e, nesta amostra, ao uso de armas. Segundo o Censo Escolar 2005 do Instituto Nacional de Estudos e Pesquisas Educacionais Anísio Teixeira, Ministério da Educação (INEP/MEC. http://www.inep.gov. $\mathrm{br} /$ basica/censo/Escolar/resultados.htm), 16\% dos estudantes de escolas públicas que terminam o ensino obrigatório não chegam a se matricular no Ensino Médio. E dos que se matricularam, $18 \%$ não concluíram os estudos, de acordo com a PNAD. Após o término do Ensino Fundamental ou quando o aluno atinge a idade de 15 anos, o adolescente é considerado apto a trabalhar sem que isto seja classificado como exploração do trabalho infantil. Essa idade coincide com a época em que o aluno termina o ensino obrigatório. A partir disso, podem surgir dificuldades em conciliar o trabalho e os estudos. A atual discussão nacional em relação à obrigatoriedade do ensino médio implica criarem-se modelos que possibilitem o sustento desses jovens para que se mantenham estudando. $\mathrm{O}$ abandono do estudo e a inserção precoce no mercado de trabalho contribuem para um afastamento do jovem do processo de socialização e inibição da impulsividade em direção a objetivos em médio prazo. Esse resultado causa maior preocupação porque a escola é um dos locais onde intervenções para reduzir o problema podem ser realizadas, e a ausência do jovem da mesma impede estratégias que poderiam ser efetivas, sobrecarregando os profissionais de saúde que devem atuar sobre o problema. Aliada a esse fato há a constatação de que um grupo significativo de jovens abandona precocemente a escola depois de sucessivos fracassos que afetam a sua auto-estima e a crença em um projeto estimulante de futuros, fato que pode estar relacionado aos resultados encontrados em relação aos transtornos psiquiátricos comuns.

Em relação aos comportamentos aditivos, a ingestão de bebidas alcoólicas aumenta a probabilidade dos adolescentes cometerem um ato violento, estando significativamente associada às duas variáveis dependentes. O uso de drogas ilícitas parece incentivar o comportamento agressivo, pois quase metade $(46,9 \%)$ dos jovens que utilizaram drogas no último mês se envolveram em uma agressão física. Esses dados referentes ao uso de álcool 17,22,26 e drogas ilícitas 21,22,27 são semelhantes aos achados de outras investigações. Em um estudo com adolescentes de 12-19 anos, realizado na Malásia, os participantes que tinham utilizado algum tipo de droga ilícita possuíam cinco vezes maior probabilidade de envolvimento em brigas quando comparados àqueles que não utilizavam drogas 22. Esses resultados são especialmente significativos pois as variáveis investigadas foram uso de álcool e drogas ilícitas e não dependência, que costumam mostrar-se associadas a crimes. Assim, o uso esporádico de drogas mostrou-se potencialmente perigoso à saúde dos jovens.

Além dos comportamentos aditivos, os problemas psicológicos se configuraram como fatores associados aos comportamentos violentos; os jovens que apresentaram transtornos mentais comuns estavam mais propensos a portar armas e a brigar. Os resultados de outras pesquisas corroboram com essa associação 17,21,22. Um estudo multicêntrico envolvendo cinco países encontrou associação entre brigas com agressão física e um sentimento de irritação e mau-humor relatado pelos jovens 17. Em outro artigo, conclui-se que os adolescentes que se sentem tristes ou inúteis por mais de duas semanas têm maior probabilidade de portar armas 22 . Dessa forma, o sofrimento psicológico transforma-se em agressão ao semelhante e à sociedade que não apresentam 
canais de desenvolvimento a esses jovens. Talvez os transtornos mentais aqui identificados sejam decorrência da situação social vivida pelo jovem e não causa da mesma.

Com base em um modelo psicossocial, tal dado evidencia a interação entre variáveis emocionais (transtornos mentais comuns) e sociais (nível sócio-econômico, possuir trabalho remunerado e estar estudando atualmente). Segundo Casiano et al. 28 , transtornos de ansiedade, uso de substâncias e controle de impulsos estão associados com comportamento violento. A impulsividade, de forma geral, pode refletir em comportamentos agressivos, uso de substâncias, e talvez implique o abando escolar e a busca por uma atividade remunerada, uma vez que o estudo implica adiar a satisfação de objetivos imediatos em função de um resultado a longo prazo. A princípio, o fumo e a prática religiosa estavam associados aos desfechos, mas perderam significância na análise ajustada; isto indica que outros fatores associados a estas variáveis são os responsáveis pela suposta relação anteriormente observada. Quanto à associação entre o porte de arma e a situação sócio-econômica, não foram encontrados artigos que suportem tal achado, entretanto Carlini-Cotrim et al. 29 encontraram em adolescentes de 15-18 anos de escolas públicas e privadas prevalências de 9,4\% e 12,2\%, respectivamente. Envolverem-se em brigas com agressão física, neste estudo, também foi mais prevalente entre os estudantes de escolas privadas $(18,4 \%)$ do que de públicas $(16,1 \%) 29$. Assim, o estabelecimento de leis mais restritivas em relação à comercialização de armas de fogo e maior fiscalização, e o estabelecimento de campanhas educativas na escola e na sociedade parecem atitudes urgentes para combater o crescimento do uso de armas e suas conseqüências nefastas. Os dois desfechos deste artigo estão inter-relacionados; os jovens que possuem algum dos comportamentos violentos têm maior probabilidade de apresentar o outro ato violento. Os participantes que brigam têm maior probabilidade de portar armas e vice-versa. Demais artigos chegaram a essa conclusão, assegurando que existe relação entre as duas condutas avaliadas 5,19,20,21,22.

Uma das maiores vantagens deste trabalho é ser o primeiro estudo nacional nessa faixa etária com delineamento transversal de base populacional, pois grande parte dos estudos encontrados refere-se a amostras de estudantes ou jovens institucionalizados, restringindo a inclusão daqueles que não fazem parte destes grupos. Em um país como o Brasil onde a taxa de abandono dos estudos é alta, o delineamento populacional permite atingir o grupo de jovens que está alijado da escola. Este estudo não possui limitações que comprometam ou alterem de forma significativa os resultados, entretanto, a interpretação dos dados é limitada devido ao seu desenho (transversal). Não pode ser feita uma relação causa-efeito entre os comportamentos agressivos estudados e as demais variáveis. Como o instrumento da pesquisa foi auto-aplicado, não é possível avaliar detalhadamente a gravidade dos comportamentos caracterizados como agressão física.

Considerado o crescimento da mortalidade entre adolescentes e o freqüente envolvimento em brigas com agressão física, o porte de arma é um problema alarmante no Brasil. Sendo assim, é fundamental uma investigação pormenorizada dos hábitos e possíveis sintomas psicossociais dos jovens envolvidos em brigas e/ou porte de arma, bem como reforçar as políticas de combate ao uso de álcool e drogas, e incentivo à permanência do jovem na escola. Além disso, é necessária a definição de estratégias articuladas a partir da escola, das organizações comunitárias e unidades básicas permitindo atingir aqueles que estão na escola e os excluídos do sistema de ensino. Cabe lembrar que os vários comportamentos de risco à saúde que foram medidos se sobrepõem, apontando a necessidade de ações integradas para enfrentá-los. 


\section{Resumo}

Objetivo do estudo foi avaliar a prevalência e fatores associados a porte de arma e envolvimento em agressão física entre adolescentes de 15 a 18 anos na cidade de Pelotas, Rio Grande do Sul, Brasil. Estudo transversal de base populacional com uma amostra representativa de 960 adolescentes entre 15 e 18 anos, residentes na cidade de Pelotas. Sortearam-se, aleatoriamente, 90 setores censitários a partir dos 448 da cidade, e foram visitados 86 domicílios de cada setor. Para análise estatística utilizou-se a regressão de Poisson. A prevalência dos comportamentos estudados na presente amostra foi de 22,8\% para participação em brigas com agressão física e 9,6\% para porte de armas nos últimos 12 meses. Conclui-se que jovens do sexo masculino que utilizam álcool elou drogas ilícitas e apresentam transtornos psiquiátricos menores mostraram maior probabilidade de portar armas e de envolverem-se em brigas com agressão física.

Armas; Agressão; Violência; Adolescente

\section{Colaboradores}

R. A. Silva fez contribuições substanciais à análise e interpretação dos dados, na revisão crítica e aprovação da versão final a ser publicada. K. Jansen fez contribuições substanciais à concepção e delineamento do estudo e à interpretação dos dados, na elaboração do artigo e aprovação da versão final a ser publicada. R. V. Godoy contribuiu de forma substancial na aquisição de dados, na elaboração do artigo e na aprovação da versão final a ser publicada. L. D. M. Souza contribuiu substancialmente na aquisição, na análise e interpretação dos dados, na revisão crítica importante para o conteúdo intelectual e na aprovação da versão final a ser publicada. B. L. Horta participou substancialmente na concepção e delineamento do estudo, na revisão crítica e aprovação da versão final a ser publicada. R. T. Pinheiro participou de forma substancial na concepção e delineamento do estudo, na revisão crítica importante para o conteúdo intelectual e na aprovação da versão final a ser publicada.

\section{Agradecimentos}

Ao Conselho Nacional de Desenvolvimento Científico e Tecnológico (CNPq).

\section{Referências}

1. Feijó RB, Oliveira EA. Comportamento de risco na adolescência. J Pediatr (Rio J.) 2001; 77:125-34.

2. Sauer MTN, Wagner MB. Acidentes de trânsito fatais e sua associação com a taxa de mortalidade infantil e adolescência. Cad Saúde Pública 2003; 19:1519-26.

3. Barros MDA, Ximenes R, Lima MLC. Mortalidade por causas externas em crianças e adolescentes: tendências de 1979 a 1995. Rev Saúde Pública 2001; 35:142-9.

4. Bergstein JM, Hemenway D, Kennedy B, Quaday S, Ander R. Guns in young hands: a survey of urban teenagers' attitudes and behaviors related to handgun violence. J Trauma 1996; 41:794-8.
5. Lowry R, Powell KE, Kann L, Collins JL, Kolbe LJ. Weapon-carrying, physical fighting, and fight-related injury among U.S. adolescents. Am J Prev Med 1998; 14:122-9.

6. Swahn MH, Bossarte RM. Gender, early alcohol use, and suicide ideation and attempts: findings from the 2005 Youth Risk Behavior Survey. J Adolesc Health 2007; 41:175-81.

7. O'Donnell L, Stueve A, Wilson-Simmons R. Aggressive behaviors in early adolescence and subsequent suicidality among urban youths. J Adolesc Health 2005; 37:517-25. 
8. Bordin IAS, Offord DR. Transtorno da conduta e comportamento anti-social. Rev Bras Psiquiatr 2000; 22:12-5.

9. Ma HK. The relation of gender-role classifications to the prosocial and antisocial behavior of chinese adolescents. J Genet Psychol 2005; 166:89-201.

10. Bor W, McGee TR, Fagan AA. Early risk factors for adolescent antisocial behaviour: an Australian longitudinal study. Aust N Z J Psychiatry 2004; 38: 365-72.

11. Habermeyer E, Herpertz SC. Dissocial personality disorder. Nervenarzt 2006; 77:605-17.

12. Farrington DP. Childhood origins of teenage antisocial behavior and adult social dysfunction. J R Soc Med 1993; 86:13-7.

13. Associação Brasileira de Institutos de Pesquisa de Mercado. Escala de indicadores para classificação socioeconômica. Rio de Janeiro: Associação Brasileira de Estudos Populacionais; 2002.

14. Mari JJ, Iacoponi E, Williams P, Simões O, Silva JB. Detection of psychiatric morbidity in the primary medical care settings in Brazil. Rev Saúde Pública 1987; 21:501-7.

15. Goldberg D, Huxley P. Common mental disorders: a bio-social model. London: Tavistock; 1992.

16. Barros AJD, Hirakata VN. Alternatives for logistic regression in cross-sectional studies: an empirical comparison of models that directly estimate the prevalence ratio. BMC Med Res Methodol 2003; 3:21.

17. Smith-Khuri E, Iachan R, Scheidt PC, Overpeck MD, Gabhainn SN, Pickett W, et al. A cross-national study of violence-related behaviors in adolescents. Arch Pediatr Adolesc Med 2004; 158:539-44.

18. Scobar-Chaves SL, Tortolero SR, Markham C, Kelder SH, Kapadia A. Violent behavior among urban youth attending alternative schools. J Sch Health 2002; 72:357-62.

19. Malek MK, Chang B, Davis TC. Fighting and weapon-carrying among seventh-grade students in Massachusetts and Louisiana. J Adolesc Health 1998; 23:94-102.
20. Durant RH, Getts AG, Cadenhead C, Woods ER. The association between weapon carrying and the use of violence among adolescents living in and round public housing. J Adolesc Health 1995; 17:376-80.

21. Alikasifoglu M, Erginoz E, Ercan O, Uysal O, Kaymak DA, Ilter O. Violent behavior among turkish high school students and correlates of physical fighting. Eur J Public Health 2004; 14:173-77.

22. Lee LK, Chen PCY, Lee KK, Kaur J. Violence-related behaviours among Malasyan adolescents: a cross sectional survey among secondary school students in Negeri Sembilan. Ann Acad Med Singapore 2007; 36:169-74.

23. Orpinas PK, Basen-Engquist K, Grunbaum JÁ, Parcel GS. The co-morbidity of violence-related behaviors with health-risk behaviors in a population of high school estudents. J Adolesc Health 1995; 16:216-25.

24. Pickett W, Craig W, Harel Y, Cumnigham J, Simpson $\mathrm{K}$, Molcho $\mathrm{M}$ et al. Cross-national study of fighting and weapon carrying as determinants of adolescent injury. Pediatrics 2005; 116:855-63.

25. Springer AE, Selwiyn BJ, Kelder SH. A descriptive sutdy of youth risk behavior in urban and rural secondary school students in El Salvador. BMC Int Health Hum Rights 2006; 6:3.

26. Análise da Pesquisa Nacional por Amostra de Domicílios - PNAD 2005. Pobreza e desigualdade. Brasília: Centro de Gestão de Estudos Estratégicos/Ministério do Trabalho e Emprego; 2007.

27. Kodjo CM, Aunger P, Ryan AS. Prevalence of, and factors associated with, adolescent physical fighting while under the influence of alcohol or drugs. J Adolesc Health 2004; 35:346.e11-6.

28. Casiano H, Belik SL, Cox BJ, Waldman JC, Sareen J. Mental disorder and threats made by noninstitutionalized people with weapons in the National Comorbidity Survey Replication. J Nerv Ment Dis 2008; 196:437-45.

29. Carlini-Cotrim B, Gazal-Carvalho C, Gouveia N. Comportamentos de saúde entre jovens estudantes das redes pública e privada da área metropolitana do Estado de São Paulo. Rev Saúde Pública 2000; 34:636-45.

Recebido em 29/Jan/2009

Versão final reapresentada em 25/Jun/2009

Aprovado em 20/Jul/2009 